\title{
Immunoregulation in Idiopathic Inflammatory Myopathies: From Dendritic Cells to Immature Regenerating Muscle Cells
}

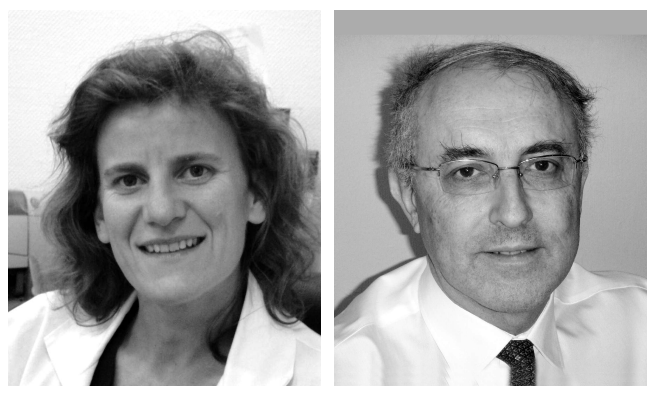

Idiopathic inflammatory myopathies (IIM) are a group of chronic muscle disorders of unknown origin that lead to muscle destruction. The original classification of IIM proposed by Bohan and Peter in 1975 including polymyositis (PM) and dermatomyositis (DM) was recently revised with the development of histopathological approaches and the discovery of myositis-specific and myositis-associated antibodies that led to the characterization of new entities. In addition to PM and adult and juvenile DM, these novel classifications distinguish inclusion-body myositis (IBM), immune-mediated necrotizing myopathy, overlap myositis, and cancer-associated myositis ${ }^{1,2}$. While distinct immunopathogenic mechanisms may occur in each subset of myositis, all IIM share a common inflammatory background associated with clinical, histological, and serological overlap. Several molecular pathways, such as adaptive and innate immune responses, autoimmunity, and nonimmune mechanisms, could influence the pathogenesis of IIM. However, the exact contribution of each one in the development of distinct phenotypes remains unclear. In this issue of The Journal, Gendek-Kubiak and Gendek highlight the contribution of dendritic cells (DC $)^{3}$. We summarize here recent insights in myositis immunoregulation from DC that are central in the development of adaptive and innate response to nonimmune mechanisms.

Dendritic cells. DC are crucial for the development of adaptive and innate immune responses. DC, subdivided into myeloid DC (mDC) and plasmacytoid DC (pDC), are present in lymphatic and blood systems and peripheral organs. They are equipped with a range of pattern-recognition receptors (PRR) and serve as sentinels of the immune system. Their activation after stimulation of PRR, such as Toll-like receptors (TLR) or C-type lectin receptors (CLR), by microbial components and/or endogenous ligands leads to their maturation and the development of an effector $\mathrm{T}$ cell immune response. In muscle biopsies from IIM, both myeloid and plasmacytoid DC have been detected with differences according to IIM subtypes. The DC present in $\mathrm{PM}$ and IBM muscle are mostly $\mathrm{mDC}$, in contrast to adult and juvenile DM, which had greater numbers of $\mathrm{pDC}$ expressing $\mathrm{CD} 4^{4,5}$. The presence of $\mathrm{pDC}$ in muscle was also increased in overlap myositis associated with anti-Jol or anti-SSA autoantibodies ${ }^{6}$. An accumulation of mature DC in muscle tissue from IIM is reported in many studies ${ }^{7,8}$, such as the study by Gendek-Kubiak and Gendek ${ }^{3}$. In their study, the authors used newly defined markers of immature and mature DC. Langerin/CD207, as CD1a previously used for the identification of immature $\mathrm{DC}^{7}$, is a marker of epidermal and epithelial Langerhans cells. Langerin is a CLR always associated with CD1a expression, and should also be useful for the identification of immature DC. Fascin is a DC protein upregulated during DC maturation that is additionally critical for antigen-presenting cell activity.

Consistent with previous studies, Gendek-Kubiak and Gendek $^{3}$ found that immature langerin DC were rarely detected compared to fascin-positive mature DC detected in all DM biopsies and in 4 out of 5 PM biopsies, without differences for the localization or the quantification of fascin-expressing DC between PM and DM. Gendek-Kubiak and Gendek ${ }^{3}$ confirm a common profile of DC subsets between PM and DM, except for a relative accumulation of more mature DC in PM than in $\mathrm{DM}^{7}$. Moreover, the presence in inflamed muscle of DC expressing fascin that is directly involved in the ability of DC to activate T cells, as well as in DC mobility and migration, highlights their local contribution in antigen presentation and $\mathrm{T}$ cell activation. Migration of DC is closely regulated during maturation through changes in chemokine receptor expression. The quasi-absence of immature DC reported by Gendek-Kubiak and Gendek ${ }^{3}$ could suggest an extramuscular maturation of DC in secondary lymphoid organs and a direct migration into muscle. However, the lack of the $\beta$ chemokines CCL19/CCL21 involved in the migration of mature DC and

See Dendritic cells in PM and DM, page 186

Personal non-commercial use only. The Journal of Rheumatology Copyright @ $\subset$ 2013. All rights reserved. 
the presence of CCL20 and its associated receptor CCR6 involved in the migration of immature DC, in association with the expression of immature CD1a-positive DC, suggest a local muscular maturation of $\mathrm{DC}^{7}$. A very similar conclusion was reached in the context of rheumatoid arthritis synovium, with differences compared to normal lymph node organization ${ }^{9}$. Such DC maturation could be enhanced by the presence in the microenvironment of proinflammatory cytokines such as interleukin 1 (IL-1) and tumor necrosis factor- $\alpha(\text { TNF- } \alpha)^{10}$. Finally, the nature of PRR expressed by DC, the type of PRR ligand, and the micro-environment in which DC are located determine the behavior of $\mathrm{DC}$ and the orientation of the immune response. Myeloid DC are potent antigen-presenting cells of adaptive immunity that stimulate $\mathrm{T}$ and $\mathrm{B}$ lymphocytes capable of highly specific response-promoting distinct phenotypes of Th cells. Plasmacytoid DC support innate immunity, in particular against viral infection, through the production of type I interferon (IFN).

$T$ cell immunity. IIM are characterized by the presence in muscle tissue of lymphocytic infiltrates consisting of CD8+ and CD4+ T cells. In PM and IBM, endomysial CD8+ T cells surround and invade muscle fibers expressing MHC class I antigens. DM is characterized by a perivascular inflammation with $\mathrm{CD} 4+\mathrm{T}$ cells, associated with capillary modifications that lead to perifascicular atrophy. The autoimmune origin of IIM is supported by a T cell-mediated myocytotoxicity and by observation of clonally expanded autoinvasive $\mathrm{T}$ cells in blood and muscle of $\mathrm{PM}^{11,12}$. Multiple and complex variables contribute to the development of naive CD4+ T cells into Th1, Th2, Th17, or Treg cells. Many studies suggest a main Th1 profile in IIM. In muscle tissue from both PM and DM, the Th1 cytokine interferon- $\gamma(\mathrm{IFN}-\gamma)$ is largely detected ${ }^{7,8,10}$, as well as IL- $12^{13}$ and IL- $18^{14}$ produced by activated DC and involved in Th1 polarization. In addition to the Th1 pathway, the presence in lymphocytic infiltrates of myositis muscle of IL-17-producing cells, as well as IL-23 produced by activated DC and involved in Th17 polarization, suggests a contribution of the IL-23-Th17 pathway ${ }^{7,8,13}$. IL-17 can act together with other proinflammatory cytokines to amplify and maintain the immune response that leads to muscle destruction ${ }^{15}$. IL-17 acts in synergy with IL-1 and TNF- $\alpha$ to induce the production by muscle cells and mononuclear cells of IL- 6 and of the $\beta$ chemokine CCL20, which in turn contribute to the differentiation and recruitment of Th17 and immature DC.

$B$ cell and humoral immunity. Humoral immunity is mediated through antibody-producing plasma cells after maturation of $\mathrm{B}$ cells following presentation of autoantigens to activated CD4+ Th cells. The autoimmune origin of IIM is supported by the presence of specific or associated myositis autoantibodies/autoantigens. Besides their use for diagnosis and classification, they also contribute to the initiation and maintenance of the immune response. In PM, $\mathrm{DM}$, and immune-mediated necrotizing myopathy, myositis-specific autoantigens such as histidyl-tRNA synthetase, $\mathrm{Mi}-2$, and HMG-CoA reductase are overexpressed in immature regenerating muscle cells also expressing high levels of HLA class I antigens ${ }^{16,17}$. Chemoattractive properties of autoantigenic aminoacyl-tRNA synthetases contribute to the migration and recruitment of $\mathrm{T}$ cells and DC. Moreover, myositis autoantibodies may act in combination with immune complexes and necrotic cells as endogenous IFN- $\alpha$ inducers ${ }^{6}$.

Innate immunity. Studies identified the contribution of the innate immune system through activation of the type I IFN system and TLR pathway in addition to the adaptive immune response ${ }^{6,13,18}$. Type I IFN-induced genes and proteins are upregulated in blood and muscle from PM and $\mathrm{DM}^{6,19}$. However, the exact source of type I IFN remains unclear because pDC, a major source of type I IFN, are rare and scattered in myositis muscle. Regenerating immature muscle fibers were identified as a local possible source of IFN- $\beta$ after activation of the TLR3 pathway and in the context of a Th1 microenvironment ${ }^{13}$. In turn, such production of type I IFN could favor HLA class I upregulation on muscle cells. Activation by endogenous ligands and self-antigens of TLR associated with nucleic acid-based pathogen-associated molecular pattern recognition may contribute to autoimmunity. In PM and DM, overexpression of TLR3 and TLR7, specifically in immature muscle fibers, has been demonstrated ${ }^{18}$. Moreover, activation of TLR3 pathways by necrotic cells, in synergy with IL-17, induces in vitro the production by muscle cells of IL- 6 and CCL20, which are involved in Th17 differentiation and migration ${ }^{18}$. In addition to TLR, CLR have been detected in inflamed muscle from PM and DM, in association with $\mathrm{mDC}$, IL-12, and IL-23 cytokines ${ }^{13}$. Those CLR-expressing cells were preferentially located around immature regenerating muscle fibers, expressing high levels of HLA class I antigens, suggesting that these muscle cells could represent a target of the immune response ${ }^{13}$.

Nonimmune mechanisms. Damaged muscle tissue caused by IIM is characterized by a degenerating process combined with muscle regeneration. However, it is unclear whether this regeneration is effective in the context of inflammation. Of note, in IIM, immature muscle fibers in charge of regeneration are characterized by the overexpression of myositis-specific autoantigens, HLA class I antigens, TLR3/7, and type I IFN ${ }^{13,16,18}$. Upregulation of HLA class I antigens on muscle fibers is a key characteristic of myositis muscle tissue. In addition to the immunological role of HLA class I molecules in the presentation of antigens, HLA class I overexpression on muscle cells may mediate muscle damage and dysfunction by itself through activation of the endoplasmic reticulum stress response pathway ${ }^{20}$. Then, the release of myositis autoantigens from damaged immature

Personal non-commercial use only. The Journal of Rheumatology Copyright @ $\subset$ 2013. All rights reserved. 
muscle cells may activate the type I IFN system when combined with necrotic cell material ${ }^{6}$, and activation of the TLR3 pathway may amplify the production of IFN- $\beta$ that in turn could contribute to HLA class I upregulation on muscle cells ${ }^{13,18}$. Focusing on these regenerating muscle fibers associated with this particular phenotype allows the formation of a self-sustaining inflammatory loop that leads to muscle wasting and defective regeneration.

\author{
ANNE TOURNADRE, $\mathrm{MD}, \mathrm{PhD}$, \\ Department of Rheumatology, \\ CHU Clermont-Ferrand, \\ Clermont-Ferrand; \\ PIERRE MIOSSEC, $\mathrm{MD}, \mathrm{PhD}$, \\ Department of Clinical Immunology and Rheumatology, \\ and Immunogenomics and Inflammation Research Unit EA 4130, \\ University of Lyon, \\ Edouard Herriot Hospital, \\ Lyon, France
}

Address correspondence to Dr. A. Tournadre, Department of Rheumatology, Gabriel Montpied Hospital, CHU Clermont-Ferrand, 58 rue Montalembert BP69, 63003 Clermont-Ferrand Cedex 1, France.

E-mail: atournadre@chu-clermontferrand.fr

\section{REFERENCES}

1. Hoogendijk JE, Amato AA, Lecky BR, Choy EH, Lundberg IE, Rose MR, et al. 119th ENMC International Workshop: Trial design in adult idiopathic inflammatory myopathies, with the exception of inclusion-body myositis. Neuromuscul Disord 2004;14:337-45.

2. Troyanov Y, Targoff IN, Tremblay JL, Goulet JR, Raymond Y, Senecal JL. Novel classification of idiopathic inflammatory myopathies based on overlap syndrome features and autoantibodies. Analysis of 100 French Canadian patients. Medicine 2005;84: 231-49.

3. Gendek-Kubiak H, Gendek EG. Fascin-expressing dendritic cells dominate in polymyositis and dermatomyositis. J Rheumatol 2012;40:186.

4. Greenberg SA, Pinkus GS, Amato AA, Pinkus JL. Myeloid dendritic cells in inclusion-body myositis and polymyositis. Muscle Nerve 2007;35:17-23.

5. Lopez de Padilla CM, Vallejo AN, McNallan KT, Vehe R, Smith $\mathrm{SA}$, Dietz AB, et al. Plasmacytoid dendritic cells in inflamed muscle of patients with juvenile dermatomyositis. Arthritis Rheum 2007;56:1658-68.

6. Eloranta ML, Barbasso Helmers S, Ulfgren AK, Rönnblom L, Alm $\mathrm{GV}$, Lundberg IE. A possible mechanism for endogenous activation of the type I interferon system in myositis patients with anti-Jo-1 or anti-Ro 52/anti-Ro 60 autoantibodies. Arthritis Rheum 2007;56:3112-24
7. Page G, Chevrel G, Miossec P. Anatomic localization of immature and mature dendritic cell subsets in dermatomyositis and polymyositis: Interaction with chemokines and Th1 cytokine-producing cell. Arthritis Rheum 2004;50:199-208.

8. Tournadre A, Porcherot M, Chérin P, Marie I, Hachulla E, Miossec $P$. Th1 and Th17 balance in inflammatory myopathies: interaction with dendritic cells and possible link with response to high-dose immunoglobulins. Cytokine 2009;46:297-301.

9. Page G, Lebecque S, Miossec P. Anatomic localization of immature and mature dendritic cells in an ectopic lymphoid organ: correlation with selective chemokine expression in rheumatoid synovium. J Immunol 2002;168:5333-41.

10. Tournadre A, Miossec P. Cytokine response in inflammatory myopathies. Curr Rheumatol Rep 2007;9:286-90.

11. Hohlfeld R, Engel AG. Coculture with autologous myotubes of cytotoxic T cells isolated from muscle in inflammatory myopathies. Ann Neurol 1991;29:498-507.

12. Benveniste $\mathrm{O}$, Chérin $\mathrm{P}$, Maisonobe $\mathrm{T}$, Merat $\mathrm{R}$, Chosidow $\mathrm{O}$, Mouthon L, et al. Severe perturbations of the blood T cell repertoire in polymyositis, but not dermatomyositis patients. J Immunol 2001;167:3521-9.

13. Tournadre A, Lenief V, Eljaafari A, Miossec P. Immature muscle precursors are a source of interferon-beta in myositis: Role of Toll-like receptor 3 activation and contribution to HLA class I up-regulation. Arthritis Rheum 2012;64:533-41.

14. Tucci M, Quatraro C, Dammacco F, Silvestris F. Interleukin-18 overexpression as a hallmark of the activity of autoimmune inflammatory myopathies. Clin Exp Immunol 2006;146:21-31.

15. Tournadre A, Miossec P. Interleukin-17 in inflammatory myopathies. Curr Rheumatol Rep 2012;14:252-6.

16. Casciola-Rosen L, Nagaraju K, Plotz P, Wang K, Levine S, Gabrielson E, et al. Enhanced autoantigen expression in regenerating muscle cells in idiopathic inflammatory myopathy. J Exp Med 2005;201:591-601.

17. Mammen AL, Chung T, Christopher-Stine L, Rosen P, Rosen A, Doering KR, et al. Autoantibodies against 3-hydroxy-3-methylglutaryl-coenzyme A reductase in patients with statin-associated autoimmune myopathy. Arthritis Rheum 2011;63:713-21.

18. Tournadre A, Lenief V, Miossec P. Expression of Toll-like receptor 3 and Toll-like receptor 7 in muscle is characteristic of inflammatory myopathy and is differentially regulated by Th1 and Th17 cytokines. Arthritis Rheum 2010;62:2144-51.

19. Walsh RJ, Kong SW, Yao Y, Jallal B, Kiener PA, Pinkus JL, et al. Type I interferon-inducible gene expression in blood is present and reflects disease activity in dermatomyositis and polymyositis. Arthritis Rheum 2007;56:3784-92.

20. Nagaraju K, Casciola-Rosen L, Lundberg I, Rawat R, Cutting S, Thapliyal R, et al. Activation of the endoplasmic reticulum stress response in autoimmune myositis: Potential role in muscle fiber damage and dysfunction. Arthritis Rheum 2005;52:1824-35.

J Rheumatol 2013;40:110-12; doi:10.3899/jrheum.121315 